\title{
Cannula Adaptations for Esophageally Fistulated Cattle
}

\author{
J.R. FORWOOD, J.L. ORTBALS, G. ZINN, AND J.A. PATERSON
}

\begin{abstract}
A modified, flexible plastisol reentrant ileal (MPI) cannula was made and tested for ability to eliminate esophageal depressions, to promote healing in fistulated steers already experiencing esophageal depressions and for general use in grazing situations. Aluminum and stainless steel 'sleeve-type' removable esophageal cannulas were coated with plastisol to reduce irritation of recently established and older esophageal fistulas. Both methods appear to reduce health problems in esophageally fistulated cattle used in grazing situations. However, lower cost of the MPI, health advantages and reduced labor requirements made it the cannula of choice over the sleeve-type cannulas.
\end{abstract}

The esophageal fistula permits collection of representative forage samples actually eaten by ruminants (Torrell 1954). However, some researchers are reluctant to employ this technique because of intensive animal maintenance and the possible mortality following surgery (Van Dyne and Torrell 1964). A variety of closure devices and esophageal cannulas have been developed, but they are usually constructed of heavy, rigid materials that irritate the lumen of the esophagus. Attempts have been made recently to develop lightweight, flexible esophageal cannulas in order to eliminate the irritation problem (Denny 1981, Ellis et al. 1984). Our experience indicates esophageal depression and irritation by heavy, rigid metal cannulas often occurs immediately following surgery and sometimes throughout the fistulated animal's life.

An 'esophageal depression', sometimes referred to as 'pocketing' (Harris et al. 1967), is an indenting or impression caused by pressure on the interior of the esophagus by the edges and upper and lower ends of metal cannula sleeves. Depressions may occur in recently established fistulas and sometimes in those which have been established for some time. They are discovered by exploring the upper and lower esophagus with the finger through the fistula. The pressure causing these depressions often results in irritation, pressure necrosis, restricted blood supply, mucosal surface erosion and accumulation of granulated and fibrous tissue and may eventually lead to nerve damage and loss of peristaltic action resulting in starvation of the animal. Rotating the cannula sleeve $180^{\circ}$ when the plug attachment is not in the center of the sleeve will sometimes eliminate pocket development by changing pressure points inside the esophagus (Harris et al. 1967).

We speculated that use of a modified flexible plastisol reentrant ileal cannula in esophageal fistulas might hold several advantages over metal cannulas. Advantages are: (1) longer sleeve length and adjustability, (2) elimination of possibility of swallowing the sleeve, (3) reduced irritation of the esophagus, (4) less possibility of accidental loss, (5) greatly reduced cost, (6) simple construction, (7) reduced labor, and (8) rapid insertion and removal. In addition, we speculated that coating metal sleeve-type cannulas with plastisol would provide a rounder, softer edge and end that may reduce the

Authors are research agronomist, USDA-ARS, Crop Production Research Unit and assistant professor, University of Missouri, Columbia 65211 ; research technician, USDA-ARS, Crop Production Research Unit, Columbia, Mo. 65211; professor, veterinary medicine, University of Missouri, Columbia 65211; and assistant professor, animal science, University of Missouri, Columbia 65211.

Contribution No. 9714. Department of Agronomy.

Manuscript accepted January 2, 1985 incidence of esophageal depressions. Our objective was to investigate the labor, cost, and health advantages, if any, of the modified cannulas when used with esophageally fistulated cattle.

\section{Materials and Methods}

\section{Plastisol Cannula}

An aluminum mold for fabrication of a modified flexible plastisol reentrant ileal cannula was made according to specifications provided by McDonnell ${ }^{1,2}$. The mold was suspended in an oven $\left(200^{\circ} \mathrm{C}\right)$ for 1 hour. When removed, the mold was immediately suspended in a thick plastisol syrup ${ }^{3}$ for 2 to 5 minutes (5-minute suspension times result in more rigid, thick cannulas). Steel wire supports (3/32 diameter) approximately $70 \mathrm{~mm}$ long were imbedded in the plastisol approximately 30 seconds after suspension begins. These were placed lengthwise on both sides where the cannula neck meets the cannula sleeve (Fig. 1) to prevent that

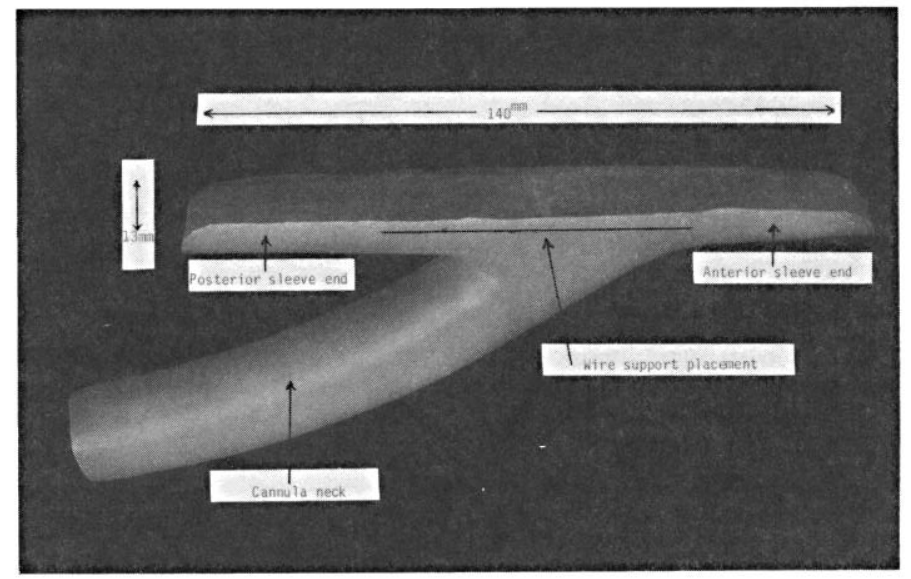

Fig. 1. Modified ileal plastisol (MPI) cannula with dimensions and labeled parts.

portion of the cannula from collapsing inward, which restricts bolus passage. After imbedding the wire, the cannula was resuspended in plastisol for the recommended time to insure coverage and to complete formation of the cannula. This step may be deleted if cannulas are immersed for 5 or more minutes, allowing them to thicken. Thick cannulas are less flexible, however, and are somewhat more difficult to insert and remove from the fistula. After suspension, excess plastisol is allowed to drop from the cannula before being re-suspended in the oven. Oven curing (at $180-200^{\circ} \mathrm{C}$ ) required 15 to 30 minutes or until the plastisol begins to smoke and develop a dark yellow or greenish color. Cooling in water was done immediately after oven removal, after which the top portion of the plastisol sleeve was slit and the cannula was pulled from the mold. Excess sleeve material was cut away and

Mike McDonnell, P.O. Box 1470, Decatur, Ill. 62525.

2Trade and company names are included for the benefit of the reader and do not imply any endorsement or preferrential treatment by the USDA of the product listed. ${ }^{3}$ Renosol Corporation, P.O. Box 8612, Ann Arbor, Mich. 48107 
smoothed leaving the sleeve a wide $U$ shape. The cannula was then similar to a double reentrant ileal cannula except for the wire supports and that the sides of the sleeve are reduced much further to allow unrestricted bolus passage (Fig. 1). A closure plug fabricated of cork, wood, or even paper toweling served to prevent forage and water loss. The cannula is rapidly inserted by firmly grasping the cannula neck and sleeve and squeezing them as close to parallel as possible. While doing this, the anterior portion of the cannula sleeve is inserted into the fistula as far as possible, allowing insertion of the posterior portion into the fistula. Then the cannula is moved slightly backward into a comfortable position with the cannula neck protruding from the fistula opening (Fig. 2). With experience, total time for insertion is $2-3$ seconds. For removal, the

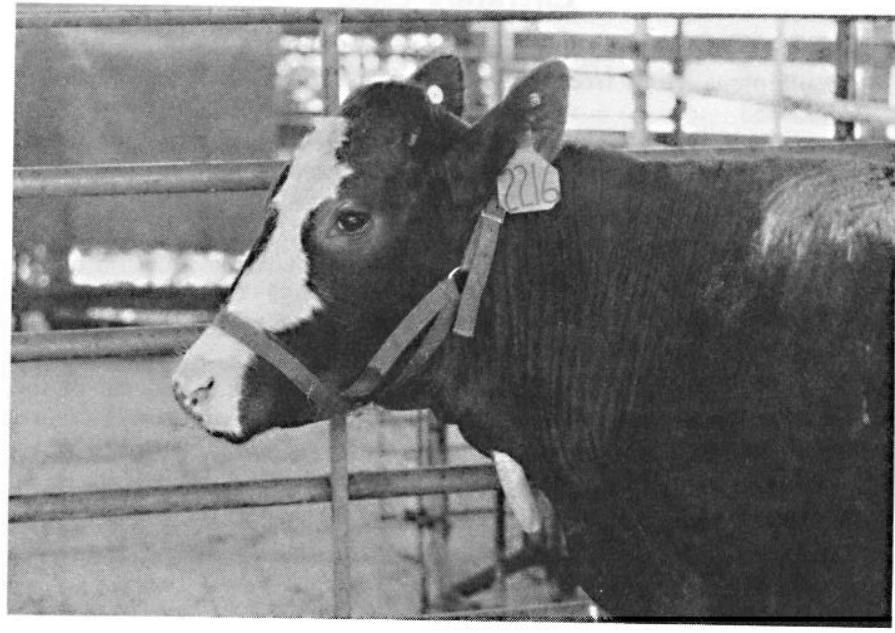

Fig. 2. Modified ileal plastisol (MPI) cannula placed in an esophageally fistulated steer.

cannula neck is grasped firmly and the cannula is moved forward until the posterior portion of the sleeve can be pulled out of the fistula. Then the cannula is moved backward and downward which results in total removal.

\section{Coating of Metal Sleeve-type Cannula}

Aluminum ${ }^{4}$ and stainless steel ${ }^{5}$ sleeve-type cannulas were suspended in an oven, heated, dipped and cured in the same manner as the cannula mold. After curing, excess plastisol around the threaded bolt and edges of the sleeve was trimmed (Fig. 3). The

${ }^{4}$ Dave Mertz, Physical Science Laboratory, Box 3548, Las Cruces, NM 88003. ${ }^{5}$ Precision Machine Co., Inc., 2933 N 36th, Lincoln, Neb. 68504.

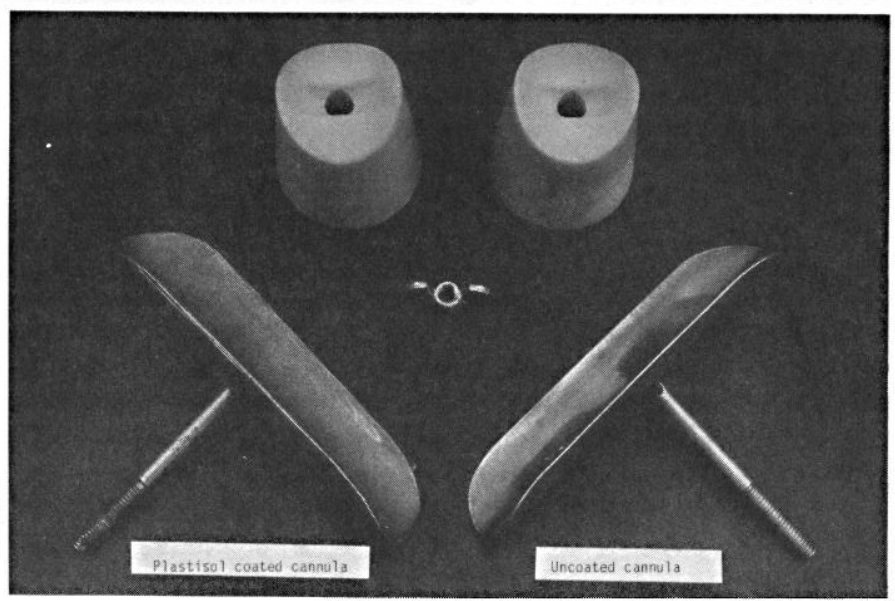

Fig. 3. Coated (left) and uncoated (right) metal sleeve-type cannulas. cannula was inspected to insure that all surfaces, especially the edges, were coated and smooth.

Five steers, approximately $181 \mathrm{~kg}$ in weight, were esophageally fistulated in March, 1983 and the same number in 1984 for use in a grazing study. At the time of surgery, removable sleeve-type metal cannulas were placed in the fistulas. The esophagus was examined every other day for esophageal depressions.

Both modified cannulas are used as closure devices during the time animals are not in use for pasture sampling. Both are completely removed for sampling purposes and re-inserted upon sampling completion to prevent loss of saliva, water and forage.

\section{Results}

Metal sleeve-type cannulas were placed in all 5 steers esophageally fistulated in 1983. Ends of the metal cannulas were alternated every other day in recently fistulated steers to avoid esophageal depressions. In spite of this, 2 steers exhibited severe depressions shortly after fistulation and before modified ileal plastisol cannulas were ready for use. When completed, the modified plastisol ileal (MPI) cannula was successful in alleviating depressions in 1 or 2 days, but apparently the damage already done was irreversible. We noted the steers were losing their ability to swallow and post-mortem examination listed starvation as the cause of death. A third steer also developed depressions shortly after fistulation, but serious health problems were avoided by the laborious process of alternating metal cannula sleeve ends every other day. Surgical placement of the fistula prevented use of an MPI cannula.

Incidence of esophageal depression in the 2 remaining steers was alleviated by constant rotating of metal cannulas. We eventually replaced their metal cannulas with MPI cannulas when esophageal depressions occurred and observed that depressions disappeared in 1 or 2 days. Metal cannulas were returned to the fistulas until depressions were noticed again, at which time MPI cannulas were inserted. This process was repeated as often as depressions occurred. Use of MPI cannulas alleviated depressions in 24 to 48 hours. Although MPI cannulas were initially used for healing, we used them successfully in these steers for extended periods of grazing.

When plastisol-coated metal cannulas were used, we found they reduced frequency and severity of esophageal depressions in recently fistulated animals, or eliminated them altogether in older animals when compared to uncoated metal cannulas. Depressions did occur, however, in animals shortly after fistulation. We have been most successful using coated cannulas after post-operative swelling has disappeared. Using uncoated metal cannulas, all 5 steers fistulated in 1984 began to show esophageal depressions 1 or 2 days after surgery. Depressions appear to be accentuated at that time due to post-operative swelling. Inserting MPI cannulas eliminated the depressions in 1 to 2 days, after which plastisol-coated metal cannulas were used. With 2 steers, it was necessary after 2 to 3 weeks to replace plastisol-coated metal cannulas with MPI cannulas several times to alleviate formation of depressions. The remaining steers used plastisol-coated metal cannulas (for about 6 weeks) until those were lost at pasture. When that occurred, MPI cannulas were inserted with no further health problems or cannula loss occurring. One steer had to be destroyed due to declining health late in the grazing season. Post-mortem examination did not reveal cause of death but did determine there were no complications due to the fistula. The remaining steers, grazing freely, fared well throughout the grazing season with no fistula complications or cannula loss. On one occasion forage became impacted around the plug, blocking the esophagus. Cutting the anterior sleeve and edges of the cannula down with a pocket knife while in the field alleviated the problem.

\section{Discussion}

The MPI cannula appears to have several advantages over metal sleeve-type cannulas in reducing health problems in fistulated 
steers. Its longer sleeve length distributes its weight on the interior esophagus over a larger area, reducing pressure on specific sites (the ends and edges) and completely covers already present depressions for extended periods, which allows healing. Its flexibility and smoothness may reduce incidence of depressions and irritation, especially when swelling occurs. The MPI cannula is labor saving in that it does not require frequent turning to avoid esophageal depression as do metal sleeve-type cannulas. It can also be modified quickly in the field and can be rapidly inserted and removed. One disadvantage may be winter use when the cannula may require soaking in hot water in order to make it flexible enough to insert.

The MPI cannula is made in one large piece, reducing the possibility of accidental loss or swallowing that accompanies some sleeve-type cannulas. Although the exposed cannula neck is fairly long, steers appear unable to pull the cannula out because the cannula neck follows the contour of the animal's neck so closely. Modified plastisol ileal cannulas are easily made and after purchasing the mold (about $\$ 35.00$ ) cost less than $\$ 1.00$ apiece. They will last a grazing season or more providing the wire supports are properly placed or the cannula is made thick enough that wire supports are not necessary.

Coating metal cannulas with plastisol also appears advantageous. Although depressions did occur, we were able to use coated cannulas without incidence of esophageal depression for longer periods of time than uncoated cannulas. We feel this is because the coating produces a round perimeter on the cannula that somewhat decreases the slow cutting action into the esophagus which produces pressure necrosis and depressions. Coated cannulas appeared to be more easily inserted and removed from the esophagus than uncoated cannulas.

From our experience, the MPI cannula appears more advantageous than coating the metal sleeve-type cannulas. Because of its health advantages, low cost and reduced labor, the MPI cannula appears useful immediately following surgery and under extended grazing conditions.

\section{Literature Cited}

Denny, G.D. 1981. A modification of an esophageal fistula plug that allows low maintenance of free-ranging sheep and goats. J. Range Manage. 34:152-153.

Ellis, W.C., E.M. Bailey, and C.A. Taylor, 1984. A silicone esophageal cannula; its surgical installation and use in research with grazing cattle, sheep or goats. J. Anim. Sci. 59:204-209.

Harris, L.E., G.P. Lofgreen, C.J. Kercher, and R.J. Raleigh. 1967. Esophageal and rumen fistula techniques for qualitative evaluation of the grazing ruminants diet. Utah Agr. Exp. Sta. Bull.

Torrell, D.T. 1954. An esophageal fistula for animal nutrition studies. J. Anim. Sci. 13:878,-884.

Van Dyne, G.M., and D.T. Torrell. 1964. Development and use of the esophageal fistula: a review. J. Range Manage. 17:7-19.

\section{RANGELAND HYDROLOGY}

by Farrel A. Branson, Gerald F. Gifford, Kenneth G. Renard, and Richard F. Hadley

Unique in its emphasis on the hydrology of rangelands, primarily arid and semiarid lands, RANGELAND HYDROLOGY provides a text for one aspect of range management where none has existed before. This expanded Second Edition presents in-depth information for those who must manage rangeland or respond to questions about the impacts of land use practices on hydrology.

Included in. the new Second Edition are a chapter on modeling with approaches to predicting the effects of land use, and a chapter on the rapidly developing field of snow pack management.

The 352-pages include 197 illustrations, providing rapid access to an assembly of data found nowhere else and useful in the preparation of environmental impact statements. Extensive bibliographic material with each chapter and a subject matter index add to the useableness of the book.

Range scientists and managers, soil conservationists, hydrologists, agricultural engineers, land reclamation specialists, wildlife managers, graduate and undergraduate students and their professors, as well as all interested in the hydrology of arid lands will find RANGELAND HYDROLOGY a valuable addition to their libraries. (352 pages paper laminated cover $\$ 15.00$ US) 